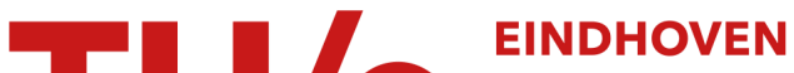 UNIVERSITY OF TECHNOLOGY
}

\section{Hall magnetocapacitance in two-dimensional electron systems}

Citation for published version (APA):

Valkering, A. M. C., Sommerfeld, P. K. H., van de Ven, R. A. M., Heijden, van der, R. W., Blom, F. A. P., Lea, M. J., \& Peeters, F. M. (1998). Hall magnetocapacitance in two-dimensional electron systems. Physical Review

Letters, 81(24), 5398-5401. https://doi.org/10.1103/PhysRevLett.81.5398

DOI:

10.1103/PhysRevLett.81.5398

Document status and date:

Published: 01/01/1998

\section{Document Version:}

Publisher's PDF, also known as Version of Record (includes final page, issue and volume numbers)

\section{Please check the document version of this publication:}

- A submitted manuscript is the version of the article upon submission and before peer-review. There can be important differences between the submitted version and the official published version of record. People interested in the research are advised to contact the author for the final version of the publication, or visit the DOI to the publisher's website.

- The final author version and the galley proof are versions of the publication after peer review.

- The final published version features the final layout of the paper including the volume, issue and page numbers.

Link to publication

\section{General rights}

Copyright and moral rights for the publications made accessible in the public portal are retained by the authors and/or other copyright owners and it is a condition of accessing publications that users recognise and abide by the legal requirements associated with these rights.

- Users may download and print one copy of any publication from the public portal for the purpose of private study or research.

- You may not further distribute the material or use it for any profit-making activity or commercial gain

- You may freely distribute the URL identifying the publication in the public portal.

If the publication is distributed under the terms of Article 25fa of the Dutch Copyright Act, indicated by the "Taverne" license above, please follow below link for the End User Agreement:

www.tue.nl/taverne

Take down policy

If you believe that this document breaches copyright please contact us at:

openaccess@tue.nl

providing details and we will investigate your claim. 


\title{
Hall Magnetocapacitance in Two-Dimensional Electron Systems
}

\author{
A. M. C. Valkering, ${ }^{1, *}$ P. K. H. Sommerfeld, ${ }^{1, \dagger}{ }^{\dagger}$ R. A. M. van de Ven, ${ }^{1}$ R. W. van der Heijden, ${ }^{1}$ \\ F. A. P. Blom, ${ }^{1}$ M. J. Lea, ${ }^{2}$ and F. M. Peeters ${ }^{3}$ \\ ${ }^{1}$ COBRA Interuniversity Research Institute, Department of Physics, Eindhoven University of Technology, \\ P.O. Box 513, NL-5600 MB Eindhoven, The Netherlands \\ ${ }^{2}$ Department of Physics, Royal Holloway, University of London, Egham, TW20 OEX, United Kingdom \\ ${ }^{3}$ Department of Physics, University of Antwerp, Universiteitsplein 1, B-2610 Antwerpen, Belgium
}

(Received 11 June 1998)

\begin{abstract}
The magnetocapacitance of a two-dimensional electron system (2DES) is investigated experimentally, both under the and away from quantum Hall conditions, at frequencies between $1 \mathrm{kHz}$ and $100 \mathrm{MHz}$. The nature of the capacitive signal in a bounded 2DES is determined by a resistive cutoff frequency $1 / \tau \propto \sigma_{x x}$, the longitudinal magnetoconductivity. A new response mechanism is reported for angular frequencies $\omega>1 / \tau$, which is controlled by the transverse or Hall conductivity $\sigma_{x y}$ and the boundaries of the sample. The mechanism is also found at frequencies far below those of the edge magnetoplasma resonances and away from the quantum Hall regime. [S0031-9007(98)07940-X]
\end{abstract}

PACS numbers: 73.40.Hm, 73.50.Jt, 73.50.Mx

The spatial dependence of the electrical properties, and, in particular, the role of the sample edges, remains one of the major issues of present-day research of twodimensional electron systems (2DES) in the quantum Hall $(\mathrm{QH})$ state [1]. A large number of experiments, employing finite-frequency methods, has recently been reported on qualitative and quantitative investigations on the current or charge distributions. Inductive techniques have been used to investigate Hall currents in the bulk of the sample [2]. Magnetocapacitance data have yielded evidence for quantum edge channels [3], but on the other hand display properties that are clearly of classical origin though they would be more readily understood in terms of quantum channels $[4,5]$. Such techniques have also been applied to test theories for composite fermions in the fractional QH effect regime [6] or to study as yet poorly understood phenomena associated with in-plane tunneling of electrons [7]. Of particular importance are direct imaging techniques of various kinds [8,9]. Very recently, novel high-resolution scanning probe imaging techniques have been developed, some of which depend basically on ac techniques [10,11]. Low-frequency $(100 \mathrm{kHz})$ ac measurements give quite different results from dc methods and are the key to imaging mobile charges [11].

Despite its increasing use, a thorough understanding of magnetocapacitance, or more generally finite-frequency properties, is still lacking. Traditionally, (magneto)capacitance is used to obtain information on the density of states in the 2DES [12]. Since it became clear that the longitudinal conductivity $\sigma_{x x}$ strongly affects the measured magnetocapacitance [13], it is now frequently used to study transport properties. Most previous experiments measured the signal between two circularly symmetric and capacitively coupled electrodes in a Corbino geometry. No signal is then obtained if $\sigma_{x x}=0$, as in the low temperature $\mathrm{QH}$ region [13]. Intuitively, $\sigma_{x x}$ is expected to be essential for capacitive coupling and consequently it has been sug- gested that "in the true quantum Hall region, it is impossible to couple capacitively to a $2 \mathrm{DE}[\mathrm{S}]$, since no charge flow can occur" [14].

Dynamical effects in strong magnetic fields for more general arrangements than the Corbino, at frequencies much lower than any characteristic frequency of the 2DES were investigated by Lea et al. [15] for the classical system of electrons on liquid helium, fully screened by metallic plates parallel to the 2DES. Independently, they were studied for the screened semiconductor 2DES by Grodnensky et al. [16]. In the experimental arrangement of Ref. [16] the magnetocapacitance varied with magnetic field by only a few percent, which could suggest that the Hall coupling is of marginal importance only. Moreover, the distinct roles of both tensor components $\sigma_{x x}$ and $\sigma_{x y}$ were not explicitly revealed. In addition, from the results of [16], it was not clear what the implications are for more conventional, low-frequency capacitance measurements (e.g., Ref. [3]).

In the present work, we demonstrate experimentally that a capacitive coupling can also arise because of charge accumulation at the edge due to the Hall effect. The charge can propagate along the edge as a result of the transverse conductivity $\sigma_{x y}$. A direct experimental proof is obtained by the use of a grounded Ohmic contact in the center of a square sample, which eliminates any conduction across the bulk of the 2DES due to finite $\sigma_{x x}$. It is shown that this new coupling mechanism is effective irrespective of the quantum Hall conditions. The relative importance of the $\sigma_{x x}$ and $\sigma_{x y}$ contributions is controlled by an $R C$ time constant $\tau=\varepsilon_{0} \varepsilon_{r} W / \sigma_{x x}$ [16], which is the time it takes excess charge to diffuse out over the entire sample with lateral dimension $W$. For angular frequencies $\omega>$ $1 / \tau$, the $\sigma_{x x}$ contribution is ineffective. The condition $\omega \tau>1$ is easily fulfilled at the $\mathrm{QH}$ regions because of the vanishing $\sigma_{x x}$. Outside the $\mathrm{QH}$ regions it is satisfied in the present work by using sufficiently high frequencies $\omega$. Our results predict that a finite residual capacitance will occur 
for $\omega \tau \gg 1$ both at and outside the $\mathrm{QH}$ regions, for the real, smooth density profile of the 2DES near the sample boundary. No explicit appeal to metallic edge channels is required.

The samples are obtained from a modulation-doped GaAs-AlGaAs heterostructure with 2DES density $n=$ $1.6 \times 10^{15} \mathrm{~m}^{-2}$ and mobility $\mu=80 \mathrm{~m}^{2} / \mathrm{V} \mathrm{s}$. Two $10 \times 10 \mathrm{~mm}^{2}$ samples were cleaved from this wafer. One was not further treated and coupled along opposite sides to two pieces of center conductor wire out of a coaxial cable, which serve as excitation and detection electrodes similar as in Ref. [16] (see inset in Fig. 1a). A $7 \times 7 \mathrm{~mm}^{2}$ mesa was etched out of the other and two Al strips were deposited along opposite sides on the remaining GaAs substrate to serve as excitation and detection electrodes. A small $\left(0.3 \times 0.3 \mathrm{~mm}^{2}\right)$ Ohmic contact was made in the center of this second sample, which is connected to ground (see inset in Fig. 1b). The rms excitation voltage $V$ was $10 \mathrm{mV}$. At low frequencies $(<100 \mathrm{kHz})$, the in- and out of phase components of the current $I$ induced in the detection electrode were measured using a current preamplifier and lock-in detector. At higher frequencies, an $\mathrm{rf}$ spectrometer was used as receiver with a $50 \Omega$ input impedance, much smaller than $1 / \omega C$, where $C \sim 10^{-12} \mathrm{~F}$ is the coupling capacitance, so that effectively the current amplitude is measured in this case. All experiments were done at a temperature of $1.5 \mathrm{~K}$, where $\sigma_{x x}$ is typically $3 \times 10^{-6} \Omega^{-1}$ at $5 \mathrm{~T}$, falling to below $10^{-8} \Omega^{-1}$ at $\nu \equiv n h / e B=2$ ( $e$ elementary charge, $B$ magnetic field, and $h$ Planck constant).
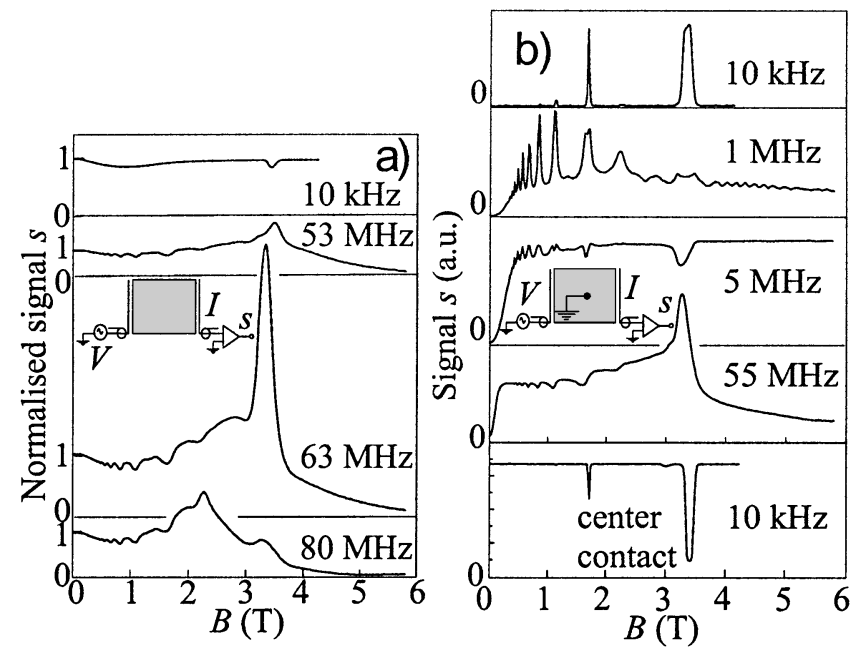

FIG. 1. (a) The experimental capacitive response normalized to the zero-field signal, at four frequencies. Inset: Experimental arrangement. The resonance at $63 \mathrm{MHz}$ coincides with the $\nu=2 \mathrm{QH}$ plateau. (b) Upper four curves: The experimental capacitive response at four frequencies with the center contact grounded. Inset: Experimental arrangement. For this sample, the resonance at $55 \mathrm{MHz}$ coincides with the $\nu=2 \mathrm{QH}$ plateau. Lowest curve: The current through the center contact at $10 \mathrm{kHz}$.
Standard resistivity data $\left(\rho_{x x}\right.$ and $\rho_{x y}$ ) were obtained from a third conventional Hall bar sample from the same wafer.

A set of data for sample 1 for four frequencies is shown in Fig. 1a. At the lowest frequency $(10 \mathrm{kHz})$, the signal is nearly field independent, but a broad shallow $(\sim 10 \%)$ minimum around $1 \mathrm{~T}$ is clearly visible in addition to a shallow dip at $\nu=2(3.3 \mathrm{~T})$. The evolution of the pattern when the frequency is increased is shown by the next three traces, which are characterized by the persistent broad minimum, followed by a series of relatively weak (10\%) Shubnikov-de Haas oscillations and a peak, which precedes a cutoff of the signal at higher fields. The data of the traces at 53,63 , and $80 \mathrm{MHz}$ are similar to those reported previously $[16,17]$. The main peak corresponds to an edge magnetoplasmon (EMP) resonance which is identified by the $1 / B$ dependence of the resonant frequency [17]. EMP's [18] are a manifestation of the Hall effect, with charge excitations localized over a distance $\ell$ near the boundary of the sample and wave velocity proportional to the Hall conductivity $\sigma_{x y} \propto 1 / B$. The damping of EMP's is determined by $\sigma_{x x}$. For the $63 \mathrm{MHz}$ trace, the frequency is chosen such that the EMP resonance coincides with the center of the $\mathrm{QH}$ region at $\nu=2$. At the other frequencies the resonance occurs outside a $\mathrm{QH}$ region, resulting in a much broader resonance. The termination of the magnetocapacitive coupling by the $\sigma_{x y}$-controlled EMP resonance suggests that the coupling is a result of the Hall effect. At zero field there is obviously no Hall effect, so then the coupling across the 2DES is purely resistive. As a result, it is expected that there is a gradual transition from purely resistive $\sigma_{x x}$ coupling at low fields to Hall $\sigma_{x y}$ coupling at high fields. The resistive coupling decreases with field because of the decrease of $\sigma_{x x}$ with field, as has previously been described in the resistive plate model [13]. The Hall coupling becomes more important at higher fields as then $\sigma_{x y} \gg \sigma_{x x}$. The minimum near $1 \mathrm{~T}$ marks the crossover between the two mechanisms.

A direct proof for the different response mechanisms is obtained by the use of sample 2 (Fig. 1b). The grounded central contact shorts out the bulk contribution, by keeping the central part of the sample at ground potential. At low frequencies, a transmission signal is observed only at the $\mathrm{QH}$ regions where $\sigma_{x x} \rightarrow 0$. When the frequency is increased, a signal appears also in between the QH regions. At high frequencies, the transmission is very similar to Fig. 1a, except at very low magnetic fields. This directly confirms the crossover from bulk to edge conduction. At low frequencies, the current through the central contact was also measured: see bottom trace in Fig. 1 b. The magnetic field dependence is complementary to that of the capacitive detector in the upper trace. The capacitive response is determined by two length scales. The first is a length $\ell=\sigma_{x x} / \varepsilon_{0} \varepsilon_{r} \omega[18,19]$ transverse to the edge, which corresponds to the distance over which charge penetrates the sample in a direction perpendicular to the edge in a time $1 / \omega$. The second is the EMP wavelength 
along the edge, given by $\lambda=\sigma_{x y} / \varepsilon_{0} \varepsilon_{r} \omega$ [18] (apart from a factor of order unity). Capacitive coupling occurs (i) via $\sigma_{x x}$ for $\ell \gg W$ (the sample size) and (ii) via $\sigma_{x y}$ for $\ell \ll W \ll \lambda$. A quantitative estimate for $\ell$ may be obtained from Fig. 1b for fields outside the $\mathrm{QH}$ regions. The frequency of $1 \mathrm{MHz}$, which clearly marks a transition regime, together with the experimental value for $\sigma_{x x}$ near $5 \mathrm{~T}$ and an average $\varepsilon_{r} \simeq 5$, yields a value $\ell \sim 6 \mathrm{~mm}$, indeed in the order of the sample dimension $W$. Values found for $\ell$ in the domain of EMP studies are in the order of $1 \mu \mathrm{m}$ [20]. In the $\mathrm{QH}$ region with $\nu=2\left(\sigma_{x x} \ll 10^{-8} \Omega^{-1}\right)$ we estimate that $\ell \ll 3 \mathrm{~mm}$ at $10 \mathrm{kHz}$, consistent with the edge mode coupling. We have discussed these effects in the context of magnetocapacitance, but they are intrinsic to the sample and would also be observed for Ohmic contacts. In the domain of EMP's, the equivalence of Ohmic or capacitive contacts was recently explicitly demonstrated [21].

In previous works on resistive coupling [13,15], it has been very helpful to visualize the physical concepts in terms of a series $C-R-C$ electrical circuit, with $R$ proportional to $1 / \sigma_{x x}$. In Fig. 2 , it is shown that also the basic features of the present data can be recovered by the circuit by adding an additional branch parallel to $R$ as shown in the insets in Figs. $2 \mathrm{c}$ and $2 \mathrm{~d}$. The parallel branch consists of a (field dependent) inductance $L(B)$ to incorporate a circuit resonance (frequency $\sqrt{2 / L C}$ ) to simulate the EMP resonance. Guided by the theory for EMP damping, the resistor $R_{1}$ in series with $L$ should be proportional to $\sigma_{x x}$. It should, however, diverge when $B \rightarrow 0$, because the Hall effect should be switched off there. The ground contact of Fig. 1b can be incorporated by splitting the resistor $R$ as in the inset in Fig. 2d. This model is very useful in understanding the experimental response, though no universal $R(B)$ and $R_{1}(B)$ dependence will fit all the data. A typical example corresponding to the configurations of Figs. 1a and $1 \mathrm{~b}$ is shown in Figs. 2c
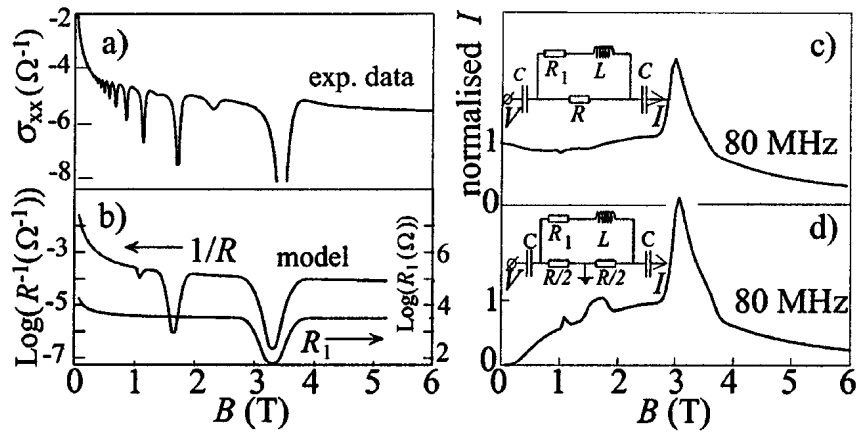

FIG. 2. (a) Experimental $\sigma_{x x}$ data (converted from measured resistivities). (b) Modeled $1 / R(B)$ and $R_{1}(B)$ dependencies, to generate the circuit [see insets in (c) and (d)] current $I$, normalized to zero field or with $R=0$, for (c) corresponding to the case without (compare Fig. 1) and (d) with (compare Fig. 2) center contact. $L(B)$ is such that the (very broad) circuit resonance $\omega=(2 / L C)^{1 / 2}$ occurs at $2.6 \mathrm{~T}$. and $2 \mathrm{~d}$, respectively, that employ models $R(B)$ and $R_{1}(B)$ displayed in Fig. 2b. The experimental $\sigma_{x x}$ trace is given in Fig. 2a.

The simulations confirm that the broad minimum near $1 \mathrm{~T}$ in the rf signal comes from the crossover between bulk (i.e., $R$-branch) and edge (i.e., $R_{1}$ - $L$-branch) contributions. The two configurations differ basically at low fields only, where the ground in the resistive path shorts the signal. For the experimental data, this implies that the edge contribution is well developed by $1 \mathrm{~T}$ at $\mathrm{rf}$ frequencies. Finally, at the frequency of $80 \mathrm{MHz}$, the (very broad) circuit resonance occurs at $2.6 \mathrm{~T}$, outside the $\mathrm{QH}$ region. Nevertheless, the peak of the signal occurs near the minimum in $\sigma_{x x}$, just as in the data.

An explicit demonstration of the existence of two coupling paths is provided by the phase behavior of the signal at intermediate frequencies, which has a very complex field dependence; an example is shown in Fig. 3a. A circuit representation is now invaluable for a qualitative understanding. Figure $3 \mathrm{~b}$ shows that the data can be nicely represented by the two-branch circuit, with a proper adjustment of the parameters (see inset). The zero crossing of the in-phase signal basically marks the crossover from the dominance of one branch to the other and occurs when all impedances $R, R_{1}$, and $1 / \omega C$ are comparable.

Away from the $\mathrm{QH}$ regions at low frequencies, the characteristic length $\ell \gg W$. Consequently, as is also clear experimentally, Figs. $1 \mathrm{~b}$ and 3 , bulk resistive coupling is also important here. On the other hand, the broad minimum near $1 \mathrm{~T}$ in Fig. 1a was interpreted as a manifestation of the $\sigma_{x y}$ coupling mechanism. At low frequencies, outside the $\mathrm{QH}$ regions, the two channels are coupled and no longer act independently.

At sufficiently low temperatures, at the $\mathrm{QH}$ regions, $\sigma_{x x}$ vanishes, and so would $\ell$. However, as is known from

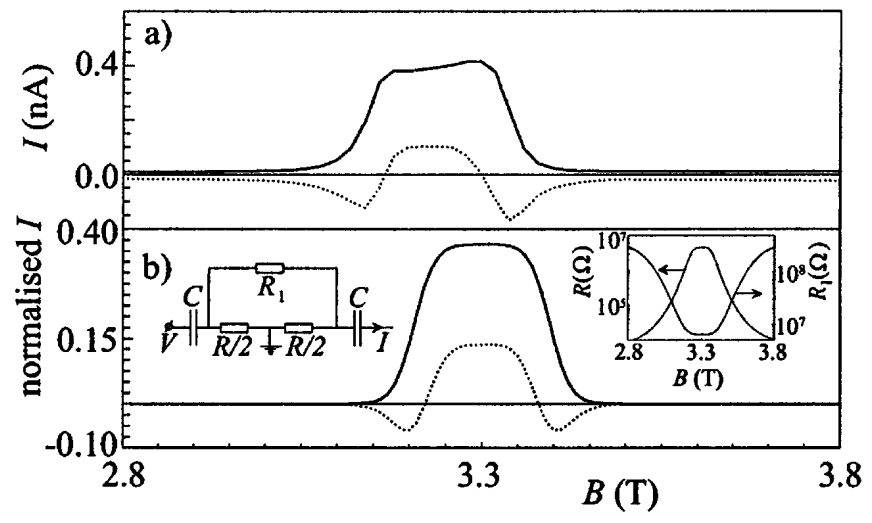

FIG. 3. (a) Experimental in- and out of phase response (dashed and solid lines, respectively) of the configuration with grounded center contact at $40 \mathrm{kHz}$. (b) Normalized in- and out of phase response (dashed and solid lines, respectively) of the circuit shown in the inset. Circuit parameters: $C=$ $2 \times 10^{-12} \mathrm{~F}, \omega / 2 \pi=40 \mathrm{kHz} ; R_{1}(B)$ and $R(B)$ : see inset. Note that no inductance has been used, as its impedance is negligible at low frequencies. 
analysis of EMP excitations the shape of the density profile near the edge is extremely important $[15,18,22]$. When $\ell$ as defined above becomes smaller than the length $\xi$ over which the equilibrium charge density decays to zero at the edge (i.e., when $\omega \tau \gg 1$ ), $\ell$ must be replaced by $\xi$ $[15,18]$. The length $\xi$ is of the order of the lateral depletion length (1 to $10 \mu \mathrm{m}$ ) and is (practically) independent of $\sigma_{x x}$ or $\sigma_{x y}$ and so of $B$. The residual capacitance at the $\mathrm{QH}$ regions observed in the experiments of Takaoka et al. [3] was taken as evidence for the existence of edge channels, whose total width is also of the order of the lateral depletion length [23]. It is important to note that such a finite residual capacitance is expected from an entirely classical analysis as well. The situation is analogous to the classical or quantum approaches of the high-frequency EMP [20]. To our knowledge a residual capacitance under non-QH conditions has not yet been observed.

From an electro-optic imaging technique employing a $5 \mathrm{kHz}$ sample potential modulation a penetration length of order $100 \mu \mathrm{m}$ was observed [9]. This length was associated with a surprisingly large and unexplained edge channel width. Possibly, this length might correspond to the classical length $\ell$ discussed in the present work $\left(\ell \sim 100 \mu \mathrm{m}\right.$ for $\sigma_{x x} \sim 10^{-10} \Omega^{-1}$ at $\left.5 \mathrm{kHz}\right)$.

In conclusion, it is shown that capacitive coupling via a 2DES can occur through the Hall effect. The coupling persists, even if $\sigma_{x x}$ is zero, provided that there is a smooth density profile near the edge, which for real systems must always be the case. The underlying principles are the same that are responsible for edge magnetoplasmons, which propagate better the smaller $\sigma_{x x}$. All observations can be understood from the local conductivities alone, without explicitly appealing to additional quantum mechanical concepts such as quantized energy levels, quantum Hall effect, or edge channels.

We thank W.C. van der Vleuten for sample growth and P. A. M. Nouwens for sample preparations.

*Present address: Fakultät für Physik, Universität Konstanz, D-78434 Konstanz, Germany.

†Present address: Technology Group, Philips Microelectronic Modules, Kreuzweg 60, D-47809 Krefeld, Germany.

[1] For a recent review of the current status of this problem, see K. Tsemekhman et al., Solid State Commun. 101, 549 (1997).
[2] E. Yahel et al., Phys. Rev. Lett. 76, 2149 (1996).

[3] S. Takaoka et al., Phys. Rev. Lett. 72, 3080 (1994); K. Oto, S. Takaoka, and K. Murase, Jpn. J. Appl. Phys. 34, 4332 (1995).

[4] W. Chen et al., Phys. Rev. Lett. 73, 146 (1994).

[5] P. K. H. Sommerfeld, R. W. van der Heijden, and F. M. Peeters, Phys. Rev. B 53, R13 250 (1996).

[6] J. S. Moon et al., Phys. Rev. Lett. 79, 4457 (1997).

[7] N. B. Zhitenev et al., Phys. Rev. Lett. 77, 1833 (1996).

[8] P.F. Fontein et al., Phys. Rev. B 43, 12090 (1991); A. J. Kent et al., Phys. Rev. Lett. 69, 1684 (1992); R. J.F. van Haren, F. A. P. Blom, and J. H. Wolter, Phys. Rev. Lett. 74, 1198 (1995); A. A. Shaskin et al., Phys. Rev. Lett. 79, 5114 (1997).

[9] W. Dietsche, K. von Klitzing, and K. Ploog, Surf. Sci. 361/362, 289 (1996).

[10] S.H. Tessmer et al., Nature (London) 392, 51 (1998).

[11] Experiments from other groups are reported and compared in B. G. Levi, Phys. Today 51, No. 4, 17 (1998).

[12] M. Kaplit and J. N. Zemel, Phys. Rev. Lett. 21, 212 (1968).

[13] R. K. Goodall, R. J. Higgins, and J. P. Harrang, Phys. Rev. B 31, 6597 (1985).

[14] Cited remark attributed to P. J. Stiles by I. M. Templeton, J. Appl. Phys. 62, 4005 (1987).

[15] M. J. Lea, A. O. Stone, and P. Fozooni, Europhys. Lett. 7, 641 (1988); M. J. Lea et al., J. Low Temp. Phys. 85, 67 (1991).

[16] I. M. Grodnenskii and A. Yu. Kamaev, JETP Lett. 52, 240 (1990); I. M. Grodnensky et al., Phys. Rev. B 44, 1946 (1991).

[17] V. A. Volkov et al., JETP Lett. 44, 655 (1987).

[18] V. A. Volkov and S. A. Mikhailov, in Modern Problems in Condensed Matter Sciences, edited by V.M. Agranovich and A. A. Maradudin (North-Holland, Amsterdam, 1991).

[19] For the screened case, a length $\ell^{\prime}$ is obtained, given by $\ell^{\prime}=\sqrt{\ell d}$, where $d$ is the distance to a screening metal plate parallel to the 2DES $[15,16]$.

[20] M. Wassermeier et al., Phys. Rev. B 41, 10287 (1990); V.I. Talyanskii et al., Phys. Rev. B 46, 12427 (1992); R. C. Ashoori et al., Phys. Rev. B 45, 3894 (1992); A. V. Polisskii et al., J. Phys. Condens. Matter 4, 3955 (1992); C. Dahl et al., Appl. Phys. Lett. 66, 2271 (1995).

[21] N. Q. Balaban et al., Phys. Rev. B 55, R13 397 (1997).

[22] S. S. Nazin and V. B. Shikin, Sov. Phys. JETP 67, 288 (1988).

[23] D. B. Chklovskii, B. I. Shklovskii, and L. I. Glazman, Phys. Rev. B 46, 4026 (1992). 